\title{
Characterization of Upper Detour Monophonic Domination Number
}

\author{
M. Mohammed Abdul Khayyoom \\ Department of Mathematics \\ PTM Govt. College, \\ Perintalmanna, Malappuram, Kerala, India. \\ khayyoom.m@gmail.com
}

\begin{abstract}
This paper introduces the concept of upper detour monophonic domination number of a graph. For a connected graph $G$ with vertex set $V(G)$, a set $M \subseteq V(G)$ is called minimal detour monophonic dominating set, if no proper subset of $M$ is a detour monophonic dominating set. The maximum cardinality among all minimal monophonic dominating sets is called upper detour monophonic domination number and is denoted by $\gamma_{d m}^{+}(G)$. For any two positive integers $p$ and $q$ with $2 \leq p \leq q$ there is a connected graph $G$ with $\gamma_{m}(G)=\gamma_{d m}(G)=p$ and $\gamma_{d m}^{+}(G)=q$. For any three positive integers $p, q, r$ with $2<p<q<r$, there is a connected graph $G$ with $m(G)=p, \gamma_{d m}(G)=q$ and $\gamma_{d m}^{+}(G)=r$. Let $p$ and $q$ be two positive integers with $2<p<q$ such that $\gamma_{d m}(G)=p$ and $\gamma_{d m}^{+}(G)=q$. Then there is a minimal DMD set whose cardinality lies between $p$ and $q$. Let $p, q$ and $r$ be any three positive integers with $2 \leq p \leq q \leq r$. Then, there exist a connected graph $G$ such that $\gamma_{d m}(G)=p, \gamma_{d m}^{+}(G)=q$ and $|V(G)|=r$.
\end{abstract}

\section{RESUMEN}

Este artículo introduce el concepto de número de dominación de desvío monofónico superior de un grafo. Para un grafo conexo $G$ con conjunto de vértices $V(G)$, un conjunto $M \subseteq V(G)$ se llama conjunto dominante de desvío monofónico minimal, si ningún subconjunto propio de $M$ es un conjunto dominante de desvío monofónico. La cardinalidad máxima entre todos los conjuntos dominantes de desvío monofónico minimales se llama número de dominación de desvío monofónico superior y se denota por $\gamma_{d m}^{+}(G)$. Para cualquier par de enteros positivos $p$ y $q$ con $2 \leq p \leq q$ existe un grafo conexo $G$ con $\gamma_{m}(G)=\gamma_{d m}(G)=p$ y $\gamma_{d m}^{+}(G)=q$. Para cualquiera tres enteros positivos $p, q, r$ con $2<p<q<r$, existe un grafo conexo $G \operatorname{con} m(G)=p, \gamma_{d m}(G)=q$ $\mathrm{y} \gamma_{d m}^{+}(G)=r$. Sean $p$ y $q$ dos enteros positivos con $2<p<q$ tales que $\gamma_{d m}(G)=p \mathrm{y}$

\section{(cc) BY-NC}

(C)2020 by the author. This open access article is licensed under a Creative Commons Attribution-NonCommercial 4.0 International License. 
$\gamma_{d m}^{+}(G)=q$. Entonces existe un conjunto DMD mínimo cuya cardinalidad se encuentra entre $p$ y $q$. Sean $p, q$ y $r$ tres enteros positivos cualquiera con $2 \leq p \leq q \leq r$. Entonces existe un grafo conexo $G$ tal que $\gamma_{d m}(G)=p, \gamma_{d m}^{+}(G)=q$ y $|V(G)|=r$.

Keywords and Phrases: Monophonic number, Domination Number, Detour monophonic number, Detour monophonic domination number, Upper detour monophonic domination number.

2020 AMS Mathematics Subject Classification: 05C69, 05C12.

\section{Introduction}

Consider an undirected connected graph $G(V, E)$ without loops or multiple edges. Let $P$ : $u_{1}, u_{2}, \ldots u_{n}$ be a path of $G$. An edge $e$ is said to be a chord of $P$ if it is the join of two non adjacent vertices of $P$. A path is said to be monophonic path if there is no chord. If $S$ is a set of vertices of $G$ such that each vertex of $G$ lies on an $u-v$ monophonic path in $G$ for some $u, v \in S$, then $S$ is called monophonic set. Monophonic number is the minimum cardinality among all the monophonic sets of $G$. It is denoted by $m(G)[1,2]$.

A vertex $v$ in a graph $G$ dominates itself and all its neighbours. A set $T$ of vertices in a graph $G$ is a dominating set if $N[T]=V(G)$. The minimum cardinality among all the dominating sets of $G$ is called domination number and is dented by $\gamma(G)[4]$. A set $T \subset V(G)$ is a monophonic dominating set of $G$ if $T$ is both monophonic set and dominating set. The monophonic domination number is the minimum cardinality among all the monophonic dominating sets of $G$ and is denoted by $\gamma_{m}(G)[5,6]$. A monophonic set $M$ in a connected graph $G$ is minimal monophonic set if no proper subset of $M$ is a monophonic set. The upper monophonic number is the maximum cardinality among all minimal monophonic sets and is denoted by $m^{+}(G)[9]$.

The shortest $x-y$ path is called geodetic path and longest $x-y$ monophonic path is called detour monophonic path. If every vertex of $G$ lies on a $x-y$ detour monophonic path in $G$ for some $x, y \in M \subseteq V(G), M$ could be identified as a detour monophonic set. The minimum cardinality among all the detour monophonic set is the detour monophonic number and is denoted by $\operatorname{dm}(G)$. A minimal detour monophonic set $D$ of a connected graph $G$ is a subset of $V(G)$ whose any proper subset is not a detour monophonic set of $G$. The maximum cardinality among all minimal detour monophonic sets is called upper detour monophonic set, denoted by $d^{+}(G)[10]$.

If $D$ is both a detour monophonic set and a dominating set, it could be a detour monophonic dominating set. The minimum cardinality among all detour monophonic dominating sets of $G$ is the detour monophonic dominating number (DMD number) and is denoted by $\gamma_{d m}(G)[7,8]$. A vertex $v$ is an extreme vertex if the sub graph induced by its neighbourhood is complete. A vertex $u$ in a connected graph $G$ is a cut-vertex of $G$, if $G-u$ is disconnected. In this article, we consider 
$G$ as a connected graph of order $n \geq 2$ if otherwise not stated. For basic notations and terminology refer [3].

Theorem 1.1 (8). Each extreme vertex of a connected graph $G$ belongs to every detour monophonic dominating set of $G$.

Example 1.1. Consider the graph $G$ given in Figure 1. Here $M_{1}=\left\{v_{1}, v_{4}\right\}$ is a monophonic set. Therefore $m(G)=2$. $M_{1}$ also dominate $G$. Hence $\gamma(G)=2$. The set $M_{2}=\left\{v_{1}, v_{2}, v_{3}\right\}$ is a minimum detour monophonic set. Thus $d m(G)=3$. $M_{2}$ does not dominate $G$. $M_{2} \cup\left\{v_{4}\right\}$ is a minimum DMD set. Therefore $\gamma_{d m}(G)=4$.

\section{UDMD Number of a Graph}

Definition 2.1. A detour monophonic dominating set $M$ in a connected graph $G$ is called minimal detour monophonic dominating set if no proper subset of $M$ is a detour monophonic dominating set. The maximum cardinality among all minimal detour monophonic dominating sets is called upper detour monophonic domination number and is denoted by $\gamma_{d m}^{+}(G)$.

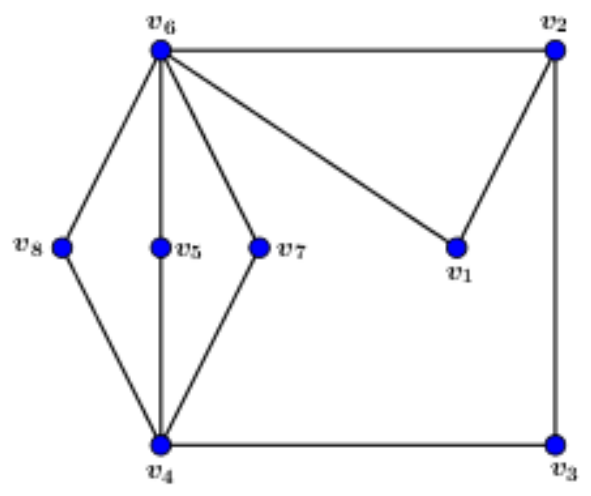

Figure 1: Graph $G$ with UDMD number 5

Example 2.1. Consider the graph $G$ given in Figure 1. The set $M=\left\{v_{1}, v_{5}, v_{6}, v_{7}, v_{8}\right\}$ is a minimal DMD set with maximum cardinality. Therefore $\gamma_{d m}^{+}(G)=5$.

Theorem 2.1. Let $G$ be a connected graph and $v$ an extreme vertex of $G$. Then $v$ belongs to every minimal detour monophonic dominating set of $G$.

Proof. Every minimal detour monophonic dominating set is a minimum detour monophonic set. Since each extreme vertex belongs to every minimum detour monophonic dominating set, the result follows. 
Theorem 2.2. Let $v$ be a cut- vertex of a connected graph $G$. If $M$ is a minimal DMD set of $G$, then each component of $G-v$ have an element of $M$.

Proof. Suppose let $A$ is a component of $G-v$ having no vertices of $M$. Let $u$ be any one of the vertex in $A$. Since $M$ is a minimal DMD set, there exist two vertices $p, q$ in $M$ such that $u$ lies on a $p-q$ detour monophonic path $P: p, u_{0}, u_{1}, \ldots, u, \ldots, u_{m}=q$ in $G$. Consider two sub-paths $P_{1}: p-u$ and $P_{2}: u-q$ of $P$. Given $v$ is a cut-vertex of $G$. Therefore both $P_{1}$ and $P_{2}$ contain $v$. Hence $P$ is not a path. This is a contradiction. That is, each component of $G-v$ have an element of every minimal DMD set.

Theorem 2.3. For a connected graph $G$ of order $n, \gamma_{d m}(G)=n$ if and only if $\gamma_{d m}^{+}(G)=n$.

Proof. First, suppose $\gamma_{d m}^{+}(G)=n$. That is $M=V(G)$ is the unique minimal DMD set of $G$, so that no proper subset of $M$ is a DMD set. Hence $M$ is the unique DMD set. Therefore $\gamma_{d m}(G)=n$. Conversely, let $\gamma_{d m}(G)=n$. Since every DMD set is a minimal DMD set, $\gamma_{d m}(G) \leq \gamma_{d m}^{+}(G)$. Therefore $\gamma_{d m}^{+}(G) \geq n$. Since $V(G)$ is the maximum DMD set, $\gamma_{d m}^{+}(G)=n$.

\section{UDMD Number of Some Standard Graphs}

\section{Example 3.1. Complete bipartite graph $K_{m, n}$}

For complete bipartite graph $G=K_{m, n}$,

$$
\gamma_{d m}^{+}(G)=\left\{\begin{array}{l}
2, \quad \text { if } \quad m=n=1 \\
n, \quad \text { if } \quad n \geq 2, m=1 \\
4, \quad \text { if } \quad m=n=3 \\
\max \{m, n\} \quad \text { if } \quad m, n \geq 2, m, n \neq 3
\end{array}\right.
$$

Proof. Case (i): Let $m=n=1$. Then $K_{m, n}=K_{2}$. Therefore $\gamma_{d m}^{+}(G)=2$.

Case (ii): Let $n \geq 2, m=1$. This graph is a rooted tree. There are $n$ end vertices. All these are extreme vertices. Therefore they belong to every DMD set and consequently every minimal DMD set.

Case (iii): If $m=n=3$, then exactly two vertices from both the particians form a minimal DMD set.

Case (iv): Let $m, n \geq 2, m, n \neq 3$. Assume that $m \leq n$. Let $A=\left\{a_{1}, a_{2}, \ldots a_{m}\right\}$ and $B=$ $\left\{b_{1}, b_{2}, \ldots b_{n}\right\}$ be the partitions of $G$. First, prove $M=B$ is a minimal DMD set. Take a vertex $a_{j}, 1 \leq j \leq m$, which lies in a detour monophonic path $b_{i} a_{j} b_{k}$ for $k \neq j$ so that $M$ is a detour monophonic set. They also dominate $G$. Hence $M$ is a DMD set. 
Next, let $S$ be any minimal DMD set such that $|S|>n$. Then $S$ contains vertices from both the sets $A$ and $B$. Since $A$ and $B$ are themselves minimal DMD sets, they do not completely belongs to $S$. Note that if $S$ contains exactly two vertices from $A$ and $B$, then it is a minimum DMD set. Thus $\gamma_{d m}^{+}(G)=n=\max \{m, n\}$.

\section{Example 3.2. Complete graph $K_{n}$}

For complete graph $G=K_{n}, \gamma_{d m}^{+}(G)=n$.

Proof. For a complete graph $G$, every vertex in $G$ is an extreme vertex. By theorem 2.1 they belong to every minimal DMD set.

\section{Example 3.3. Cycle graph $C_{n}$}

For Cycle graph $G=C_{n}$ with $n$ vertices ,

$$
\gamma_{d m}^{+}(G)=\left\{\begin{array}{l}
3, \quad \text { if } n \leq 7, n \neq 4 \\
2, \quad \text { if } n=4 \\
4+\frac{n-7-r}{3}, \quad \text { if } n \geq 8, n-7 \equiv \bmod (3)
\end{array}\right.
$$

Proof. For $n \leq 7$ the results are trivial. For $n \geq 8$, let $C_{n}: v_{1}, v_{2}, v_{3}, \ldots, v_{n}, v_{1}$ be the cycle with $n$ vertices. Then the set of vertices $\left\{v_{1}, v_{3}, v_{n-1}\right\}$ is a minimal detour monophonic set but not dominating. This set dominates only seven vertices. There are $n-7$ remaining vertices. If $r$ is the reminder when $n-7$ is divided by 3 , then $\frac{n-7-r}{3}+1$ vertices dominate the remaining vertices. Therefore every minimal DMD set contains $4+\frac{n-7-r}{3}$ vertices.

\section{Characterization of $\gamma_{d m}^{+}(G)$}

Theorem 4.1. For any two positive integers $p$ and $q$ with $2 \leq p \leq q$ there is a connected graph $G$ with $\gamma_{m}(G)=\gamma_{d m}(G)=p$ and $\gamma_{d m}^{+}(G)=q$.

Proof. Construct a graph $G$ as follows. Let $C_{6}: u_{1}, u_{2}, u_{3}, u_{4}, u_{5}, u_{6}, u_{1}$ be the cycle of order 6 . Join $p-1$ disjoint vertices $M_{1}=\left\{x_{1}, x_{2}, \ldots, x_{p-1}\right\}$ with the vertex $u_{1}$. Let $M_{2}=\left\{y_{1}, y_{2}, \ldots, y_{q-p-1}\right\}$ be a set of $q-p-1$ disjoint vertices. Add each vertex in $M_{2}$ with $u_{4}$ and $u_{6}$. Let $x_{p-1}$ be adjacent with $u_{2}$ and $u_{6}$. This is the graph $G$ given in Figure 2.

Since all vertices except $x_{p-1}$ in $M_{1}$ are extreme, they belong to every minimum monophonic dominating set and DMD set. The set $M=M_{1} \cup\left\{u_{4}\right\}$ is a minimum monophonic dominating set. Therefore $\gamma_{m}(G)=p$. Moreover, the set of all vertices in $M$ form a DMD set and is minimum. That is $\gamma_{d m}(G)=p$. 
Next, we prove that $\gamma_{d m}^{+}(G)=q$. Clearly $N=M_{1} \cup M_{2} \cup\left\{u_{5}, u_{6}\right\}$ is a DMD set. $N$ is also a minimal DMD set of $G$. For the proof, let $N^{\prime}$ be any proper subset of $N$. Then there exists at least one vertex $u \in N$ and $u \notin N^{\prime}$. If $u=y_{i}$, for $1 \leq i \leq q-p-1$, then $y_{i}$ does not lie on any $x-y$ detour monophonic path for some $x, y \in N^{\prime}$. Similarly if $u \in\left\{u_{5}, u_{6}, x_{p-1}\right\}$, then that vertex does not lie on any detour monophonic path in $N^{\prime}$. Thus $N$ is a minimal DMD set. Therefore $\gamma_{d m}^{+}(G) \geq q$.

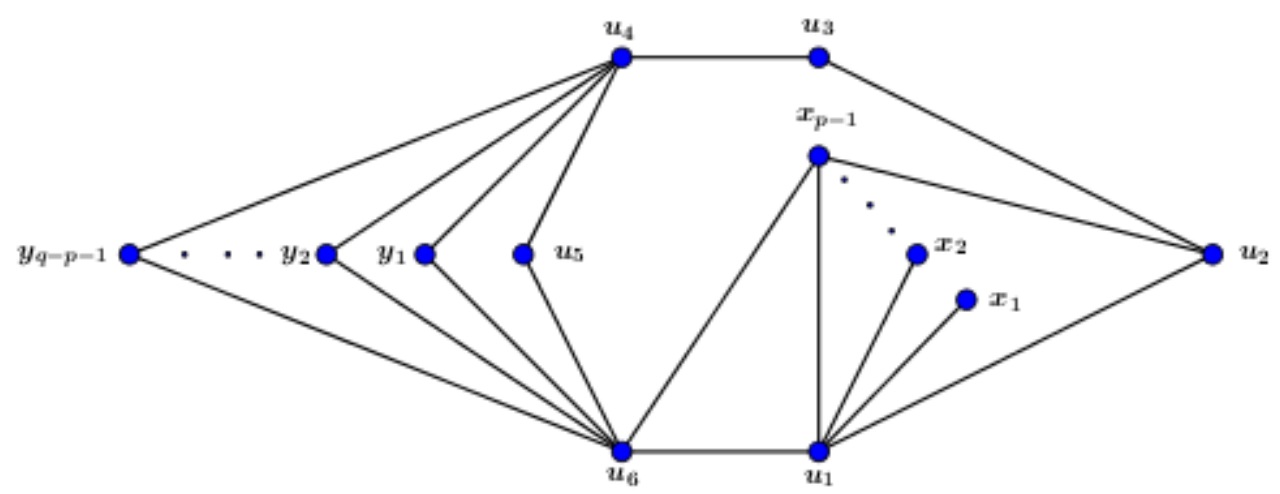

Figure 2: $\gamma_{m}(G)=\gamma_{d m}(G)=p$ and $\gamma_{d m}^{+}(G)=q$.

Note that $N$ is a minimal DMD set with maximum cardinality. On the contrary, suppose there exists a minimal DMD set, say $T$, whose cardinality is strictly greater than $q$. Then there is a vertex $u \in T, u \notin N$. Therefore $u \in\left\{u_{2}, u_{3}, u_{4}\right\}$. If $u=u_{4}$, then $M_{1} \cup\left\{u_{4}\right\}$ is a DMD set properly contained in $T$ which is a contradiction. If $u=u_{3}$, then the set $M_{1} \cup\left\{u_{3}, u_{5}\right\}$ is a DMD set which is a proper subset of $T$ and is a contradiction. If $u=u_{2}$, then the set $\left(N-\left\{u_{6}\right\}\right) \cup\left\{u_{2}\right\}$ is a DMD set properly contained in $T$ and is a contradiction. Thus $\gamma_{d m}^{+}(G)=q$.

Theorem 4.2. For any three positive integers $p, q, r$ with $2<p<q<r$, there is a connected graph $G$ with $m(G)=p, \gamma_{d m}(G)=q$ and $\gamma_{d m}^{+}(G)=r$.

Proof. Let $G$ be the graph constructed as follows. Take $q-p$ copies of a cycle of order 5 with each cycle $C_{i}$ has a vertex set $\left\{d_{i}, e_{i}, f_{i}, g_{i}, h_{i}\right\}$, for $1 \leq i \leq q-p$. Join each $e_{i}$ with all other vertices in $C_{i}$. Also join the vertex $f_{i-1}$ of $C_{i-1}$ with the vertex $d_{i}$ of $C_{i}$. Let $\{u, v\}$ and $\left\{b_{1}, b_{2}, \ldots, b_{r-q+1}\right\}$ be two sets of mutually non adjacent vertices. Join each $b_{i}$ with $u$ and $v$, for $1 \leq i \leq r-q+1$. Join another $p-2$ pendent vertices with $u$ and one pendent vertex with $d_{1}$. This is the graph $G$ given in Figure 3.

The set $M_{1}=\left\{a_{0}, a_{1}, a_{2} \ldots, a_{p-2}\right\}$ is the set of all extreme vertices and belongs to every monophonic dominating set and DMD set (Theorem 1.1). Clearly $M_{1}$ is not monophonic. But $M_{1} \cup\{v\}$ is a monophonic set and is minimum. Therefore $m(G)=p$. Take $M_{2}=\left\{e_{1}, e_{2}, \ldots, e_{q-p}\right\}$. Then $M_{1} \cup M_{2} \cup\{v\}$ is a DMD set and is minimum. Therefore $\gamma_{d m}(G)=p-1+q-p+1=q$. 


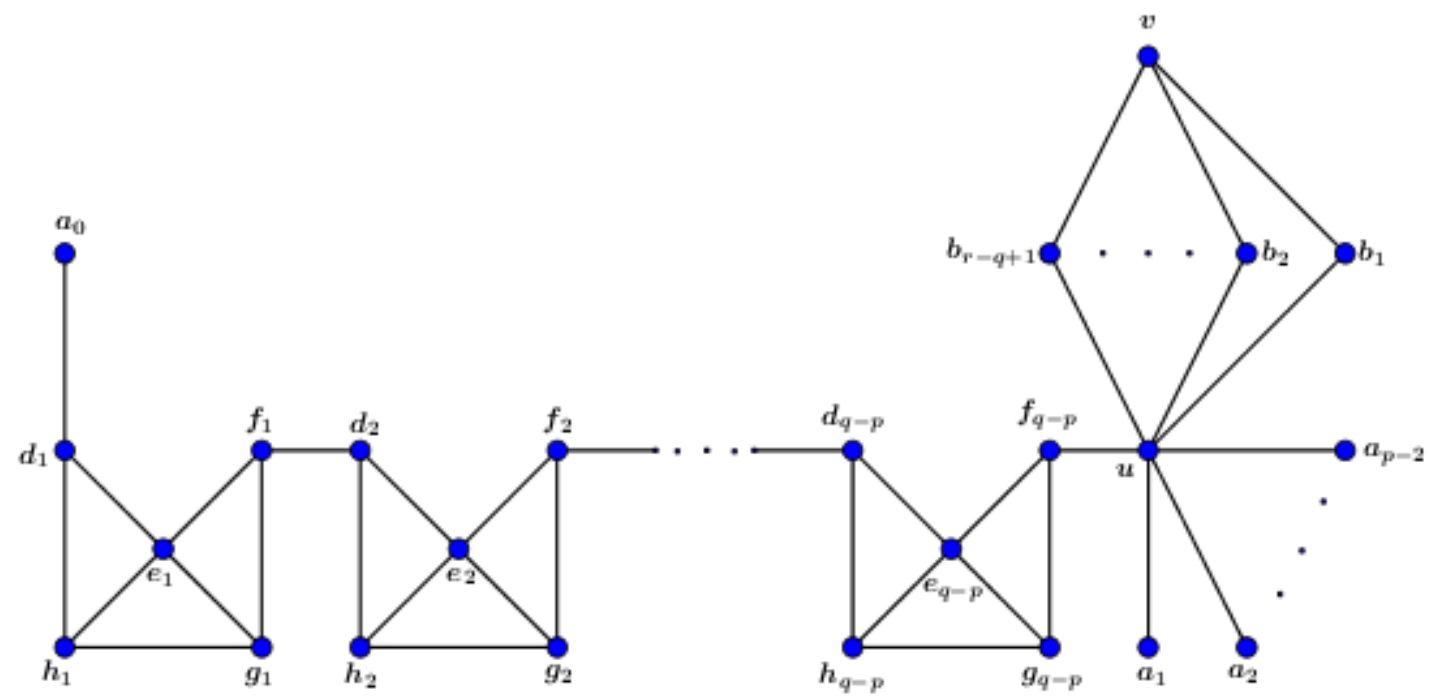

Figure 3: Graph $G$ with $m(G)=p, \gamma_{d m}(G)=q$ and $\gamma_{d m}^{+}(G)=r$.

Let $M_{3}=\left\{b_{1}, b_{2}, \ldots, b_{r-q+1}\right\}$. Then $M=M_{1} \cup M_{2} \cup M_{3}$ is a DMD set. Now $M$ is a minimal DMD set. On the contrary, suppose $N$ is any proper DMD subset of $M$ so that there exists at least one vertex in $M$ which does not belong to $N$. Let $u \in M$ and $u \notin N$. Clearly $u \notin M_{1}$ since $M_{1}$ is the set of all extreme vertices. If $u=e_{i}$ for some $i$, then the vertex $e_{i}$ does not belong to any detour monophonic path induced by $N$. Therefore $u \notin M_{2}$. Similarly $u \notin M_{3}$. This is a contradiction. Hence $M$ is a minimal DMD set with maximum cardinality. Therefore $\gamma_{d m}^{+}(G)=\left|M_{1}\right|+\left|M_{2}\right|+\left|M_{3}\right|=(p-1)+(q-p)+(r-q+1)=r$.

Theorem 4.3. Let $p$ and $q$ be two positive integers with $2<p<q$ such that $\gamma_{d m}(G)=p$ and $\gamma_{d m}^{+}(G)=q$. Then there is a minimal DMD set whose cardinality lies between $p$ and $q$.

Proof. Consider three sets of mutually disjoint vertices $M_{1}=\left\{a_{1}, a_{2}, \ldots, a_{q-n+1}\right\}, M_{2}=\left\{b_{1}, b_{2}, \ldots, b_{n-p+1}\right\}$ and $M_{3}=\{x, y, z\}$. Join each vertex $a_{i}$ with $x$ and $z$ and each vertex $b_{j}$ with $y$ and $z$. Add $p-2$ pendent vertices $M_{4}=\left\{c_{1}, c_{2}, \ldots, c_{p-2}\right\}$ with the vertex $y$. This is the graph $G$ given in Figure 4 . Since $M_{4}$ is the set of all extreme vertices, it belongs to every DMD set. But $M_{4}$ is not a DMD set. The set $M=M_{4} \cup\{x, z\}$ is a minimum DMD set. Therefore $\gamma_{d m}(G)=p$.

Consider the set $N=M_{1} \cup M_{2} \cup M_{4}$. We claim $N$ is a minimal DMD set with maximum cardinality. On the contrary, suppose there is a set $N^{\prime} \subset N$ which is a DMD set of $G$. Then there exists at least one vertex, say $u$ in $N$ which does not belong to $N^{\prime}$. Clearly $u \notin M_{4}$ since it is the set of all extreme vertices. If $u \in M_{1}$, then $u=a_{i}$ for some $i$. Then the vertex $a_{i}$ does not lie on any detour monophonic path, which is a contradiction. Similarly, if $u \in M_{2}$, we get a contradiction. Thus $N$ is a minimal DMD set. Therefore $\gamma_{d m}^{+}(G) \geq q$. 


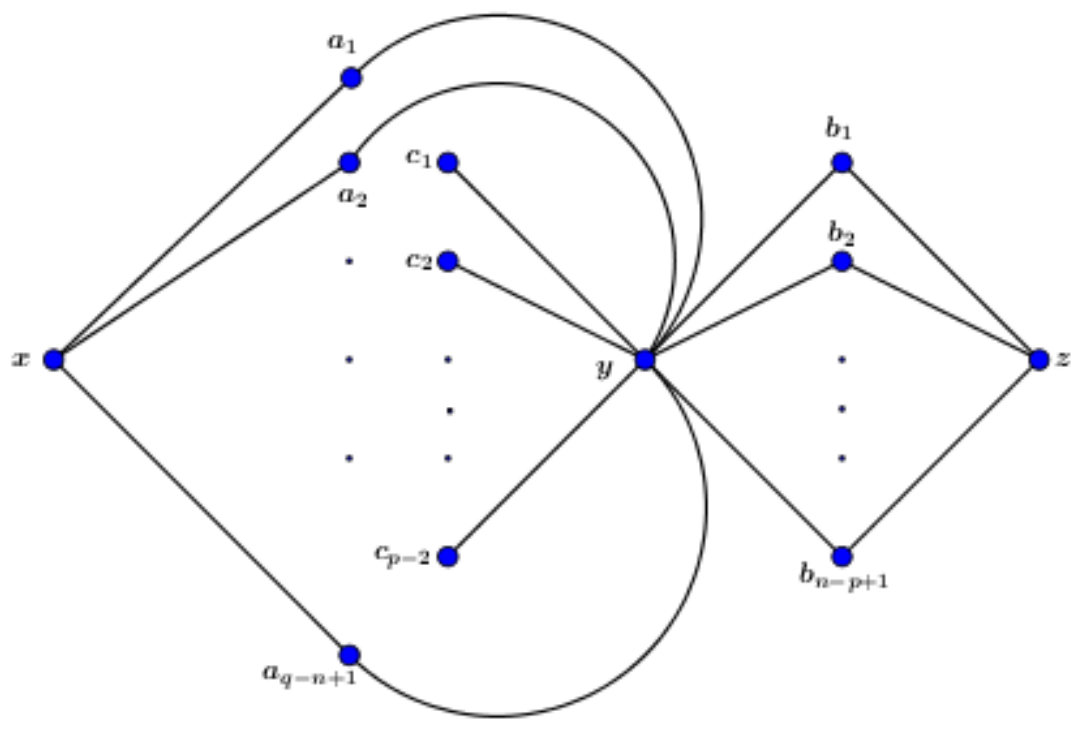

Figure 4: Graph $G$ with $\gamma_{d m}(G)=p$ and $\gamma_{d m}^{+}(G)=q$

Next, we claim that $N$ has the maximum cardinality of any minimal DMD set. If $\gamma_{d m}^{+}(G)>q$, there is at least one vertex $v \in V(G), v \notin N$ and belongs to a minimal DMD set. Therefore $v \in M_{3}$. If $v=x$, then the set $M_{2} \cup M_{4} \cup\{v\}$ is a minimal DMD set having less than $q$ vertices. Similarly if $v=z$, then the set $M_{1} \cup M_{4} \cup\{v\}$ is a minimal DMD set. For $v=y$, the set $N \cup\{y\}$ is not a minimal DMD set. Therefore $\gamma_{d m}^{+}(G) \leq q$.

Let $n$ be any number which lies between $p$ and $q$. Then there is a minimal DMD set of cardinality $n$. For the proof, consider the set $T=M_{2} \cup M_{4} \cup\{x\}$. T is a minimal DMD set. If $T$ is not a minimal DMD set, there is a proper subset $T^{\prime}$ of $T$ such that $T^{\prime}$ is a minimal DMD set. Let $u \in T$ and $u \notin T^{\prime}$. Since each vertex in $M_{4}$ is an extreme vertex, $v \notin M_{4}$. If $u=x$, then the vertex $u$ is not an internal vertex of any detour monophonic path in $T^{\prime}$. A similar argument may be made if $u \in M_{2}$. This leads to a contradiction. Therefore $T$ is a minimal DMD set with cardinality $(n-p+1)+(p-2)+1=n$.

Theorem 4.4. Let $p, q$ and $r$ be any three positive integers with $2 \leq p \leq q \leq r$. Then, there exists a connected graph $G$ such that $\gamma_{d m}(G)=p, \gamma_{d m}^{+}(G)=q$ and $|V(G)|=r$.

Proof. Let $K_{1, p}$ is a star graph with leaves set $M_{1}=\left\{u_{1}, u_{2}, \ldots, u_{p}\right\}$ and let $u$ be the support vertex of $K_{1, p}$. Insert $r-q-1$ vertices $M_{2}=\left\{v_{1}, v_{2}, \ldots, v_{r-q-1}\right\}$ in the edges $u u_{i}$ respectively for $1 \leq i \leq r-q-1$. Add $q-p$ vertices $M_{3}=\left\{x_{1}, x_{2}, \ldots, x_{q-p}\right\}$ with this graph and join each $x_{i}$ with $u$ and $u_{1}$. This is the graph $G$ as shown in Figure 5. Here $|V(G)|=(q-p)+p+(r-q-1)+1=r$. The length of a detour monophonic path is 4 . 


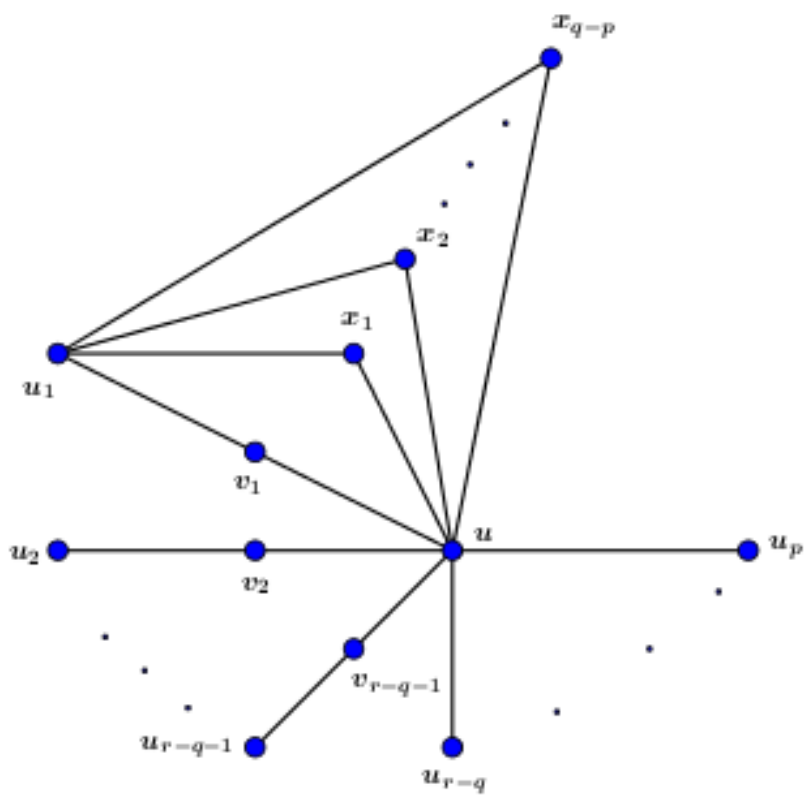

Figure 5: Graph $G$ with $\gamma_{d m}(G)=p$ and $\gamma_{d m}^{+}(G)=q$

Let $T=M_{1}-\left\{u_{1}\right\}$. All the vertices in $T$ are extreme vertices and belong to all DMD sets and minimal DMD sets. Clearly $M_{1}$ is a DMD set with minimum cardinality. Therefore $\gamma_{d m}(G)=p$. Let $N=T \cup M_{3} \cup\left\{v_{1}\right\}$. Then $|N|=(p-1)+(q-p)+1=q$. We claim that $N$ is a minimal DMD set with maximum cardinality.

On the contrary, suppose there is a proper subset $N^{\prime}$ of $N$ which is a minimal DMD set of $G$. Then there exists at least one vertex $x \in N, x \notin N^{\prime}$. Clearly $x \notin T$. If $x \in M_{3}$, then $x=x_{i}$ for some $i, 1 \leq i \leq q-p$. Then the vertex $x_{i}$ does not lie on any $u-v$ detour monophonic path for $u, v \in N^{\prime}$. If $x=v_{1}$ then $v_{1}$ does not lies on any detour monophonic path in $N^{\prime}$. Thus no such vertex $x$ exists. This is a contradiction. Therefore $\gamma_{d m}^{+}(G) \geq q$.

To prove maximum cardinality of $N$, suppose there exists a minimal DMD set $S$ with $|S|>q$. Since $S$ contains $T$, the set of all extreme vertices, the vertex $x$ lies on some $u-v$ detour monophonic path for all $x \in\left\{u, v_{2}, v_{3}, . ., v_{r-q-1}\right\}$. Now $S$ is a minimal DMD set having more than $q$ vertices and $u, v_{2}, v_{3}, \ldots, v_{r-q-1} \notin S$. Therefore $S=\left\{v_{1}\right\} \cup M_{3} \cup\left\{u_{1}\right\} \cup T$. Then $N$ is properly contained in $S$. This is a contradiction. Therefore $\gamma_{d m}^{+}(G)=q$. Hence the proof. 


\section{References}

[1] P. A. P. Sudhahar, M. M. A. Khayyoom and A. Sadiquali, "Edge Monophonic Domination Number of Graphs". J.Adv.in Mathematics, vol. 11, no. 10, pp. 5781-5785, 2016.

[2] P. A. P. Sudhahar, M. M. A. Khayyoom and A. Sadiquali, "The Connected Edge Monophonic Domination Number of Graphs". Int. J Comp.Applications, vol. 145, no. 12, pp. 18-21, 2016.

[3] G. Chartrand and P. Zhang, Introduction to Graph Theory. MacGraw Hill, 2005.

[4] T. W. Haynes, S. T. Hedetniemi and P. J. Slater, Fundementals of Domination in Graphs. 208, Marcel Dekker Inc, New York, 1998.

[5] J. Jhon and P. A. P. Sudhahar, "On The Edge Monophonic Number of a Graph Filomat", vol. 26, no. 6, pp. 1081-1089, 2012.

[6] J.Jhon and P.Arul Paul Sudhahar, "The Monophonic Domination Number of a Graph, Proceedings of the International Conference on Mathematics and Business Managment", pp. 142-145, 2012 .

[7] M. M. A. Khayyoom and P. A. P. Sudhahar. "Edge Detour Monophonic Domination Number of a Graph. International Journal of Pure and Applied Mathematics", vol. 120, no. 7, pp. 195-203, 2018.

[8] M. M. A. Khayyoom and P. A. P. Sudhahar, "Connected Detour Monophonic Domination Number of a Graph". Global Journal of Pure and Applied Mathematics, vol. 13, no. 5, pp. 241-249, 2017.

[9] S. R. Chellathurai, and S. Padma Vijaya, "Upper Geodetic Domination Number of a Graph" Int. Journal of Cont. Math Sci., vol. 10, no. 1, pp. 23-36, 2015.

[10] P. Titus, A. P. Santhakumaran, K. Ganesamoorthy, "Upper Detour Monophonic Number of a Graph", Electronic Note in Discrete Mathematics, vol. 53, pp. 331-342, 2016. 